\title{
Thermal behavior of Quantum Cellular Automaton wires
}

\author{
C. Ungarelli*, S. Francaviglia, M. Macucci, G. Iannaccone \\ Dipartimento di Ingegneria dell'Informazione, Università di Pisa \\ Via Diotisalvi, 2, I-56126 Pisa, Italy
}

(June 24, 2018)

\begin{abstract}
We investigate the effect of a finite temperature on the behavior of logic circuits based on the principle of Quantum Cellular Automata (QCA) and of ground state computation. In particular, we focus on the error probability for a wire of QCA cells that propagates a logic state. A numerical model and an analytical, more approximate, model are presented for the evaluation of the partition function of such a system and, consequently, of the desired probabilities. We compare the results of the two models, assessing the limits of validity of the analytical approach, and provide estimates for the maximum operating temperature.
\end{abstract}

Typeset using REVTEX

${ }^{*}$ Current address: School of Mathematical Sciences, University of Portsmouth, Mercantile House, Hampshire Terrace, Portsmouth P01 2EG, UK 


\section{INTRODUCTION}

In recent years a new paradigm for computation has been proposed by Craig Lent and coworkers [1], based on the concept of Quantum Cellular Automata (QCA). Such a concept, although extremely difficult to implement from a technological point of view [2], has several interesting features that make it worth pursuing. The basic building block is made up of a single cell, containing two electrons that can be localized in four different areas or "dots," located at the vertices of a square, as shown in each of the cells represented in Fig. 1(a). Coulomb repulsion forces the two electrons to occupy dots that are aligned along one of the diagonals, and each of the two possible alignments is associated with a logic state. By placing cells next to each other, a wire can be formed (binary wire), along which polarization enforced at one end will propagate [3], as a consequence of the system of charges relaxing down to the ground state. We can see this also as the logic state of the first cell propagating down the chain until it reaches the last cell. It has been shown that, by properly assembling two-dimensional arrays of cells, it is possible to implement any combinatorial logic function [3]. The basic principle of operation of such circuits is therefore the relaxation of the system to the ground state, thus leading to the often used expression "ground-state computation".

Even in the case of perfectly symmetric and identical cells, the configuration of the QCA circuit may depart from the ground state as a consequence of thermal excitations. If the energy separation between the ground state and the first few excited states is small, their occupancy will be nonnegligible even at low temperatures, and the logic output may be corrupted. A complete understanding of the behavior of QCA arrays as a function of temperature is thus essential for any practical application of the QCA concept. The problem of errors due to finite temperature operation was first addressed by Lent [G], on the basis of entropy considerations. Our approach consists in a detailed study of thermal statistics for QCA arrays, retrieving the results of Ref. [4] as a special case, and allowing treatment of cells with more than just two states. We have developed both a numerical model, which enables us to study relatively short chains made up of six-state cells in full detail, and an 
analytical model, which can be used for arbitrarily long chains of two-state cells. In both cases, we have considered a semiclassical approximation, and computed the probability of the system being in the ground state and that of presenting the correct logic output, i.e. of having the last cell of the chain in the expected logic state. Since only one configuration

corresponds to the ground state, while several different configurations are characterized by the correct logic output, the probability of having the correct output is always larger than that of being exactly in the ground state.

In Sec. [1] we present the cell model we have considered for both approaches and the semi-classical approximation that we have chosen to adopt. We also discuss the structure of the energy spectrum for the excited states of a chain of cells. In Sec. III we present the procedure that has been followed for the calculation of the partition function with the numerical method and the associated results for the probabilities of correct operation as a function of temperature. The analytical model is described in Sec. $\square$, together with the associated results and a comparison with those from the numerical model.

\section{MODEL}

Our approach is semi-classical insofar as electrons are treated as classical particles, with the only additional property that they can tunnel between dots belonging to the same cell. This is a reasonable approximation if the tunneling matrix elements between the dots of a cell are small enough to strongly localize the electrons, which therefore behave as well defined particles.

Our model chains are characterized by two geometrical parameters: $d$ is the distance between neighboring cell centers, $a$ is the distance between two dots in a cell. We represent the driver cell, i.e. the cell whose polarization state is externally enforced, with bold lines and indicating only the electron positions (see Fig. 1(a)); driven cells are represented with solid lines and each dot is indicated with a solid circle if occupied or with an empty circle otherwise. Within each cell we consider a uniformly distributed $(e / 2$ per dot, where $e$ 
is the electron charge) positive background charge, which makes each cell overall neutral and prevents anomalous behaviors in the nearby cells, due to the uncompensated monopole component of the electrostatic field. In particular, the repulsive action of the uncompensated electrons in a driver cell can "push" the electrons in the nearby driven cell away, thus leading to the formation of an unwanted state in which electrons are aligned along the side further from the driver cell.

In our calculations we have considered the GaAs/AlGaAs material system and assumed a uniform relative permittivity of 12.9: this is a reasonable approximation, since the permittivity of AlGaAs does not differ significantly from that of the GaAs layer, where the electrons are confined. For this study we have neglected, for the sake of simplicity and of generality, the effects of the semiconductor-air interface and of the metal gates defining the dots, whose rigorous treatment would have required considering a specific layout [5].

For silicon-on-insulator QCA cells [6], materials with quite different permittivities come into play: silicon, silicon oxide and air, but reasonable estimates could be obtained by repeating our calculations with a relative permittivity corresponding to that of silicon oxide, since most of the electric field lines are confined in the oxide region embedding the silicon dots. Moreover, estimates of the performance obtained with this approximation would be conservative, since part of the field lines are actually in the air over the device, whose relative permittivity is unitary, thus leading to a stronger electrostatic interaction and therefore to a reduced importance of thermal fluctuations.

As we have already stated, the two minimum energy configurations of a cell are those with the electrons aligned along one of the diagonals, since these correspond to the maximum separation between the electrons. However, other configurations are also possible, and, depending on intercell spacing, they can appear in the first few excited states of a binary wire. We consider all of the six configurations that can be assumed by two electrons in four dots, excluding only those with both electrons in the same dot, which correspond to too large an energy.

We define the two lowest energy configurations (those with the electrons along the di- 
agonals) state 1 and state 0 as indicated in Fig. 1 (b), while the corresponding polarization values are 1 and -1 , respectively. Polarization values are defined [7] as

$$
P=\frac{Q_{1}+Q_{3}-Q_{2}-Q_{4}}{2}
$$

where $Q_{i}$ is the charge in the $i$-th dot, with the first dot being at the top right and the others numbered counterclockwise. Configurations with the two electrons along one of the four sides of the cell have higher energies, as stated before, and do not correspond to a well defined logic state. For this reason, we define them as $X$ states.

The energy is computed as the electrostatic energy of a classical system of charges [8]:

$$
E=\sum_{i \neq j} \frac{q_{i} q_{j}}{4 \pi \epsilon_{0} \epsilon_{r} r_{i j}}
$$

Since in our model the total charge in each dot is either the background charge (empty dot) or the algebraic sum of the background charge and the charge of an electron, it can take on only two values: $+e / 2$ or $-e / 2$, which implies that

$$
q_{i} q_{j}=\frac{1}{4} e^{2} \operatorname{sgn}\left(q_{i} q_{j}\right)
$$

If we write the interelectronic distance $r_{i j}$ in terms of the ratio $R=d / a$ and of the configuration, the energy of a binary wire can be written as

$$
E=\frac{e^{2}}{4 a} \frac{1}{4 \pi \epsilon_{0} \epsilon_{r}} \sum_{i \neq j} \frac{s_{i j}}{\sqrt{\left(n_{i j} R+l_{i j}\right)^{2}+m_{i j}^{2}}},
$$

where $n_{i j} \in\left\{0, \ldots, N_{\text {cell }}-1\right\}$ is the number of cells between the cell containing dot $i$ and the cell containing dot $j, s_{i j} \in\{-1,1\}$ indicates the sign of $q_{i} q_{j}, l_{i j} \in\{-1,0,1\}$ and $m_{i j} \in\{0,1\}$, indicate the position of dots $i$ and $j$ inside the corresponding cells. In particular, $l_{i j}$ is equal to 0 if both dots $i$ and $j$ are on the left side or on the right side of the cell, to -1 if dot $i$ is on the right side and $\operatorname{dot} j$ is on the left side and to 1 if dot $i$ is on the left side and dot $j$ is on the right one. Furthermore, $m_{i j}$ is equal to 0 if both dots $i$ and $j$ are on the top or on the bottom of a cell, to 1 if one dot is on the top and the other is on the bottom.

We have considered a binary wire made up of six cells (one of which is a driver cell in a fixed polarization state) with size $a=40 \mathrm{~nm}$ and computed the energy values corresponding 
to all possible $6^{5}$ configurations. The values thus obtained have been ordered with the purpose of studying the energy spectra for different parameter choices. Let us define $R=$ $d / a$. If $R \gg 1$, i.e. $d \gg a$, the interaction between neighboring cells is substantially due to just the dipole component, and a discrete spectrum is observed already for $R=2.5$ (see Fig. (2), with clear steps: the ground state corresponds to configurations with all cells in the same logic state: either all 1 or 0 ; the first excited state, for $R=2.5$, includes configurations with one "kink," i.e. with one cell flipped with respect to the rest of the chain. Higher steps correspond to a larger number of kinks. Energy values are expressed with reference to the ground state energy and in kelvin, i.e. as the result of the division of the actual energies in joule by the Boltzmann constant.

If $R$ is decreased, the interaction between neighboring cells is incremented and made more complex, so that $X$ states do appear, as shown in Fig. 2 for $R=1.75$. The various plateaus start merging and a continuous spectrum is approached. In particular, if we decrease $R$ while keeping a constant, and thereby reducing the separation between neighboring cells, the difference between the energy of the ground state and that of the first excited state is expected to increase as $1 / R$, due to the increased electrostatic interaction. However, this is true only down to a threshold value of $R$, below which the splitting between the first excited state and the ground state starts decreasing, as shown in Fig. 3, where the energy split is plotted as a function of $R$ for a cell size $a$ of $40 \mathrm{~nm}$, for a wire with 2 (dotted line), 3 (dashed line), and 6 (solid line) cells. This sudden change of behavior can be understood on the basis of the previously discussed results: below the threshold value for $R$, the configuration for the first excited state contains a cell in the $X$ state, thereby disrupting the operation of the wire and lowering the splitting between the first excited state and the ground state.

In the inset of Fig. 3 we report the dependence of the splitting between the two lowest energy states on the number of cells. These results are for $R=2.5$, i.e. for a condition in which no $X$ state appears. Once the number of cells is larger than a few units, the splitting quickly saturates to a constant value. This is easily understood if we consider that the first excited state is characterized by the cell at the end of the wire being polarized opposite to 
the others: the strength of the electrostatic interaction drops quickly along the chain, and hence no significant change is determined by the addition of cells beyond the first five or six. The energy splitting has been computed for a cell size $a=40 \mathrm{~nm}$ and, as can be deduced from Eq.(4), is inversely proportional to $a$. It can thus be increased by scaling down cell dimensions.

\section{NUMERICAL RESULTS FOR THE THERMAL BEHAVIOR}

In order to compute the probabilities, at a finite temperature, for the various configurations, we introduce the partition function of the wire:

$$
Z=\sum_{i} e^{-\beta E_{i}}
$$

where $E_{i}$ is the energy of the $i$-th configuration and $\beta=k T, k$ being the Boltzmann constant and $T$ the temperature. The summation is performed over all configurations with the first cell in a given input logic state. The probability $P_{\mathrm{gs}}$ of the entire system being in the ground state can be evaluated by taking the ratio of the Boltzmann factor for the ground state to the partition function:

$$
P_{\mathrm{gs}}=\frac{e^{-\beta E_{\mathrm{gs}}}}{Z}=\frac{1}{1+\sum_{i \neq \mathrm{gs}} e^{-\beta \Delta E_{i}}},
$$

where $\Delta E_{i}=E_{i}-E_{\mathrm{gs}}$, and the sum extends over all excited states.

As already mentioned in the introduction, $P_{\mathrm{gs}}$ is not the only quantity of interest. From the point of view of applications, we are mainly interested in knowing the probability $P_{\text {clo }}$ of obtaining the correct logic output, which is higher than $P_{\mathrm{gs}}$, because several configurations, besides the ground state, exhibit the correct polarization for the output cell (the cell at the end of the chain). We can compute $P_{\text {clo }}$ by summing over the probabilities corresponding to all such configurations, that we label with the subscript $j$ :

$$
P_{\text {clo }}=\frac{\sum_{j} e^{-\beta E_{j}}}{Z}
$$


We have computed both $P_{\mathrm{gs}}$ and $P_{\text {clo }}$ as a function of the ratio of the splitting $\Delta E$ between ground state and first excited state to $k T$. The results for a chain of 6 cells are presented in Fig. 6 : in the limit $\Delta E /(k T) \ll 1$ all the configurations (a total of $6^{N-1}, N$ being the number of cells) become equally probable and the probability $P_{\mathrm{gs}}$ reaches its minimum value $1 / 6^{N-1}$. The probability of correct logic output, instead, reaches a minimum value of $1 / 6$, as a consequence of the six possible states of the output cell being equally probable.

It should be noted that an error probability of a few percent may appear unacceptable for any practical circuit application, but data readout must always be done via some detector [5], which is characterized by a time constant necessarily longer than the typical settling time of the QCA circuit. Therefore, each reading will be the result of an averaging procedure, and will be compared to a threshold value. In such a case, an error probability of a few percent for the output state will lead in most cases to a vanishingly small error probability for the actual output of the readout circuit.

As already noted, the number of possible configurations for a circuit with $N$ cells (one of which is assumed to be the driver cell and hence in a given, fixed configuration) is $6^{N-1}$. Thus the CPU time required to explore all such configurations grows exponentially with the number of cells, which limits the length of the binary wires that can be investigated with this approach in a reasonable time down to about ten cells. In order to assess the thermal behavior of long wires, we have developed the approximate analytical approach that will be described in the next section.

\section{ANALYTICAL MODEL}

The development of an analytical model for the investigation of the thermal behavior of a QCA chain requires a main simplifying assumption, in order to make the algebraic treatment possible: for each cell we consider only two configurations, the ones corresponding to the logic states 1 and 0 , and, thus, to polarization +1 and -1 . From the discussion in Sec. प it is apparent that the larger $R$, the better this approximation is, because the role of the $X$ 
states is reduced.

Let us consider a generic 1-dimensional chain consisting of $N$ cells and introduce the following 1-dimensional Ising Hamiltonian

$$
\mathcal{H}=-J \sum_{i=1}^{N-1} \sigma_{i} \sigma_{i+1},
$$

where for each cell labeled by the index $i$ the variable $\sigma_{i}$ corresponds to the polarization and therefore assumes the two values \pm 1 , and the positive quantity $J$ (which has the dimension of an energy) is related to the splitting $\Delta E$ between the ground state and the first excited state energies of an $N$-cell system by $J=\Delta E / 2$. Let us point out that there is a twofold degeneracy of the ground state, corresponding to the two configurations $\left\{\sigma_{i}=1, \forall i\right\}$ and $\left\{\sigma_{i}=-1, \forall i\right\}$. This degeneracy is removed by enforcing the polarization state of the driver cell, which corresponds to enforcing the configuration of one of the boundary sites; our conventional choice is $\sigma_{1}=1$. In this case, the lowest energy state corresponds to the configuration $\left\{\sigma_{i}=1, \forall i\right\}$. The partition function of the $N$-cell system described by the Hamiltonian (8) with the boundary condition $\sigma_{1}=1$ is given, in analogy with Eq.(5), by the following expression:

$$
Z=\sum_{\{\sigma\}} e^{-\beta \mathcal{H}}
$$

where $\{\sigma\}$ stands for the summation over all possible states, i.e. $\left\{\sigma_{1}=1, \sigma_{i}= \pm 1, i=\right.$ $2 \ldots N\}$. This last expression can be written as

$$
Z=\sum_{\sigma_{2} \ldots \sigma_{N}} V\left(1, \sigma_{2}\right) V\left(\sigma_{2}, \sigma_{3}\right) \ldots V\left(\sigma_{N-1}, \sigma_{N}\right)
$$

where $V\left(\sigma, \sigma^{\prime}\right)=e^{\beta \sigma \sigma^{\prime}}$. In order to compute the r.h.s. of Eq. (10), the usual procedure consists in introducing the transfer matrix [9]

$$
\mathcal{V}=\left(\begin{array}{cc}
e^{\beta J} & e^{-\beta J} \\
e^{-\beta J} & e^{\beta J}
\end{array}\right),
$$

whose eigenvalues are 


$$
\lambda_{+}=e^{\beta J}+e^{-\beta J}, \quad \lambda_{-}=e^{\beta J}-e^{-\beta J} .
$$

The expression for the matrix $\mathcal{V}^{N-1}$ is given by

$$
\mathcal{V}^{N-1}=\left(\begin{array}{ll}
\frac{\lambda_{+}^{N-1}+\lambda_{-}^{N-1}}{2} & \frac{\lambda_{+}^{N-1}-\lambda_{-}^{N-1}}{2} \\
\frac{\lambda_{+}^{N-1}-\lambda_{-}^{N-1}}{2} & \frac{\lambda_{+}^{N-1}+\lambda_{-}^{N-1}}{2}
\end{array}\right)
$$

It then follows that the partition function (10) reads

$$
Z=\left[\mathcal{V}^{N-1}\right]_{11}+\left[\mathcal{V}^{N-1}\right]_{12}=\left(e^{\beta J}+e^{-\beta J}\right)^{N-1},
$$

where the subscripts indicate specific elements of the $\mathcal{V}^{N-1}$ matrix. This explicit formula for the partition function allows us to derive an analytical expression for the probability of the system being in its ground state as a function of the temperature and of the energy splitting between the two lowest states. Since the ground state energy for the Hamiltonian (8) is $E_{\mathrm{gs}}=-J(N-1)$, we obtain

$$
P_{\mathrm{gs}}=\frac{e^{-\beta E_{\mathrm{gs}}}}{Z}=\frac{e^{\beta J(N-1)}}{Z}=\frac{1}{\left(1+e^{-\beta \Delta E}\right)^{N-1}} .
$$

Finally, we can derive an analytical expression also for the probability of obtaining the correct logic output, in analogy with what we have already done in the numerical case. We need to determine the occupation probability of a generic state with $\sigma_{1}=\sigma_{N}=1$, which corresponds to having the correct output, because the polarization of the $N$-th cell (output cell) is the same as that of the first cell. To this purpose, we evaluate the following "reduced" partition function:

$$
Z_{R}=\sum_{\sigma_{2} \ldots \sigma_{N-1}} V\left(1, \sigma_{2}\right) V\left(\sigma_{2}, \sigma_{1}\right) \ldots V\left(\sigma_{N-1}, 1\right)
$$

where again $V\left(\sigma, \sigma^{\prime}\right)=e^{\beta \sigma \sigma^{\prime}}$. Using the transfer matrix (11), it follows that $Z_{R}=\left[\mathcal{V}^{N-1}\right]_{11}$, and hence

$$
P_{\text {clo }}=\frac{Z_{R}}{Z}=\frac{\left[\mathcal{V}^{N-1}\right]_{11}}{\left[\mathcal{V}^{N-1}\right]_{11}+\left[\mathcal{V}^{N-1}\right]_{12}}=\frac{1}{2}\left[1+(\tanh (\beta \Delta E / 2))^{N-1}\right]
$$

The above derived analytical expressions have been used to compute $P_{\text {gs }}$ and $P_{\text {clo }}$ as a function of temperature for a chain of 6 cells, cell size $a=40 \mathrm{~nm}$, cell separation $d=$ 
$100 \mathrm{~nm}$. Results are presented with dashed lines in Fig. 5, together with those obtained with the numerical technique (solid lines). For temperatures below about $2 \mathrm{~K}$ (those for which reasonably low error probabilities can be achieved) the analytical model provides values that are in almost perfect agreement with those from the more detailed numerical approach. The situation differs at higher temperatures, because higher energy configurations, containing cells in $X$ states, start being occupied and are properly handled by the numerical model, while they are not at all included in the analytical approach. In particular, while for large values of the temperature the numerical $P_{\text {clo }}$ tends to $1 / 6$, as previously discussed, the analytical $P_{\text {clo }}$ approaches the value $1 / 2$, because the output cell can be in one of two states with the same probability. Analogous considerations can be made for $P_{\mathrm{gs}}$, which becomes extremely small $\left(1 / 6^{5}\right)$ for higher temperatures in the numerical case, while drops just to $1 / 2^{5}$ in the analytical case, since there are $2^{5}$ possible configurations.

In Fig. 6, $P_{\text {clo }}$ and $P_{\mathrm{gs}}$ are reported as a function of the ratio $\Delta E /(k T)$ (with a semilogarithmic scale) for the analytical model (thick solid line), and for the numerical model with $R=2$ (thin solid line), $R=2.5$ (dashed line), and $R=4$ (dotted line). As expected, the agreement improves with increasing $R$, because of the reduced relevance of the $X$ states. For $\Delta E /(k T)$ of the order of a few units, the error probability becomes very small and the analytical expression can be reliably used to evaluate it.

In particular, the analytical expression allows us to provide estimates of the maximum operating temperature for a QCA chain formed by a given number of cells. We have computed the maximum operating temperature allowing a given correct logic output probability, as a function of the number of cells: results are reported in Fig. 7 for $P_{\text {clo }}=0.6$ (solid line), 0.9 (dashed line), 0.99 (dotted line) and cell size $a=40 \mathrm{~nm}$, intercell separation $d=100 \mathrm{~nm}$. The maximum operating temperature, for a number of cells above a few tens, drops logarithmically, which leads to a linear behavior in the logarithmic representation of Fig. 7 . 


\section{CONCLUSIONS}

We have developed both a numerical and an analytical approach to the investigation of the thermal dependence of QCA wire operation. Both methods are based on a semiclassical approach, in which electrons are considered as classical particles interacting via the Coulomb force, with, however, the possibility of tunneling between the quantum dots belonging to the same cell. The electrostatic energy associated with each configuration has been evaluated and used for the calculation of the occupancies, via the partition function.

Numerical results have been derived for wires with six-state cells, which realistically reproduce the behavior of QCA systems, provided that the confinement in each quantum dot is strong enough. The numerical procedure thus developed is general and is currently being applied to the investigation of thermal limitations for simple logic gates, including the effect of spurious, $X$ states.

The analytical approach has allowed a detailed analysis of the error probability due to thermal excitations in arbitrarily long wires, generalizing the findings of previous studies, and the possibility of extending it to selected basic gates is being investigated.

It is clear from our results that the operating temperature depends on the ratio of the energy splitting $\Delta E$ to $k T$. It could therefore be raised by increasing $\Delta E$, which means reducing the dielectric permittivity or scaling down cell dimensions. As already mentioned, the silicon-on-insulator material system offers better perspectives of higher-temperature operation, due to the lower permittivity of silicon oxide. However, scaling down in any semiconductor implementation is limited by the increasing precision requirements [2], therefore a trade-off between manufacturability and operating temperature has to be accepted. Implementations at the molecular level could provide better opportunities, due to the reduced dimensions, but their actual feasibility is still being assessed. 


\section{ACKNOWLEDGMENTS}

This work has been supported by the ESPRIT Project N. 28667 ANSWERS, Autonomous Nanoelectronic Systems With Extended Replication and Signalling. 


\section{REFERENCES}

[1] C. S. Lent, P. D. Tougaw, and W. Porod, Appl. Phys. Lett. 62, 714 (1993).

[2] M. Governale, M. Macucci, G. Iannaccone, C. Ungarelli, J. Martorell, J. Appl. Phys., 85, 2962 (1999).

[3] P. D. Tougaw and C. S. Lent, J. Appl. Phys. 75, 1818 (1994).

[4] C. S. Lent, P. D. Tougaw, and W. Porod, in "The Proceedings of the Workshop on Physics and Computing," Nov. 17-20, 1994, Dallas, TX, p. 1.

[5] G. Iannaccone, C. Ungarelli, M. Macucci, E. Amirante, M. Governale, Thin solid Films, 336, 145 (1998).

[6] F. E. Prins, C. Single, F. Zhou, H. Heidemeyer, D. P. Kern, E. Plies, Nanotechnology, 10, $132(1999)$.

[7] M. Girlanda, M. Governale, M. Macucci, G. Iannaccone, Appl. Phys. Lett., 75, 3198 (1999).

[8] J. D. Jackson, Classical Electrodynamics (Wiley, New York, 1962), p. 20.

[9] See e.g. J. R. Baxter, Exactly solved models in statistical mechanics (Academic Press, London, 1982). 


\section{FIGURES}

FIG. 1. a) Chain of 6 QCA cells, with the indication of the cell size $a$ and of the intercell separation $d$; b) representation of the six possible states of a cell with two electrons.

FIG. 2. Energy spectrum and cell configurations for a six-cell wire, for two values of the ratio $R$ of intercell separation $d$ to cell size $a$, with $a=40 \mathrm{~nm}$.

FIG. 3. Energy split between the first excited state and the ground state for a chain of 2 (dotted line), 3 (dashed line), 6 (solid line) cells as a function of the ratio $R$ of the intercell separation $d$ to the cell size $a$, assumed to be $40 \mathrm{~nm}$. The energy split as a function of the number of cells is reported in the inset, for a value $R=2.5$.

FIG. 4. Plot of the probability of correct logic output $P_{\text {clo }}$ and of the probability of ground state occupancy $P_{\mathrm{gs}}$ for a chain of six cells, with $\mathrm{R}=2.5$, as a function of the ratio $\Delta E /(k T)$

FIG. 5. Plot of the probability of correct logic output $P_{\text {clo }}$ and of the probability of ground state occupancy $P_{\mathrm{gs}}$ for a chain of six cells as a function of temperature, computed with the numerical six-state model (solid lines) and with the analytical two-state model (dashed lines). The cell size $a$ is $40 \mathrm{~nm}$ and the intercell separation $d$ is $100 \mathrm{~nm}$.

FIG. 6. Plot of $P_{\text {clo }}$ and $P_{\text {gs }}$ as a function of $\Delta E /(k T)$ for the analytical two-state model (thick solid line) and for the numerical six-state model with $R=2$ (thin solid line), $R=2.5$ (dashed line), and $R=4$ (dotted line).

FIG. 7. Maximum operating temperature of a QCA binary wire as a function of the number of cells, for obtaining a probability of correct logic output 0.6 (solid line), 0.9 (dashed line), and 0.99 (dotted line). The cell size is $40 \mathrm{~nm}$ and the intercell separation is $100 \mathrm{~nm}$. 
a)

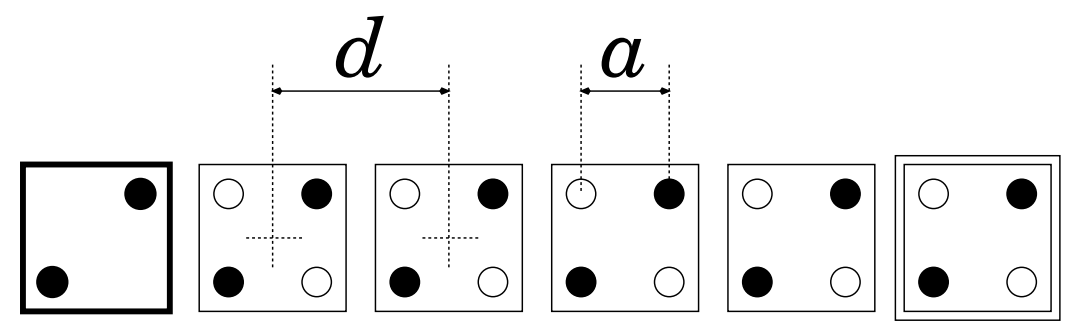

b) $\underbrace{\begin{array}{ll}\hline & \bullet \\ \bullet & \circ\end{array} \quad \begin{array}{ll}\bullet & 0 \\ \circ & \bullet\end{array}}_{\text {state } 1}$

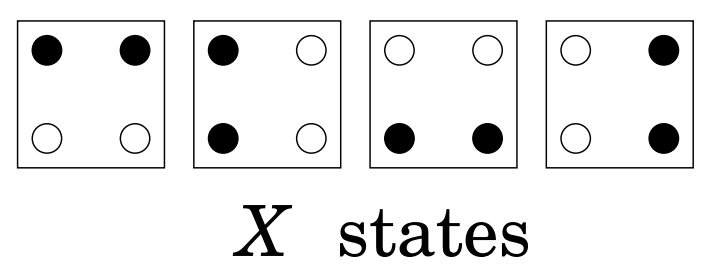

Fig. 1

C. Ungarelli et al.

J. Appl. Phys. 


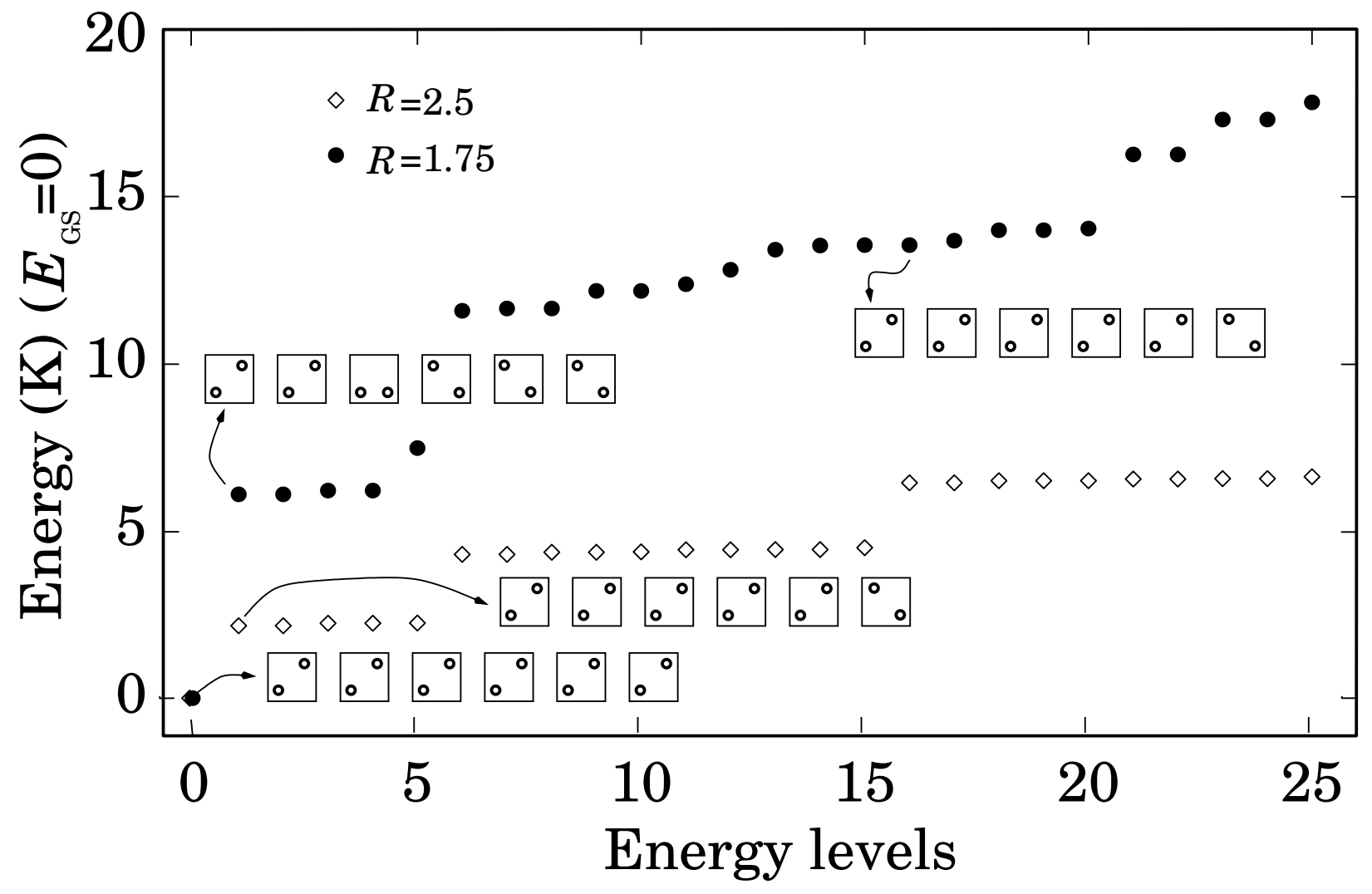

Fig. 2

C. Ungarelli et al.

J. Appl. Phys. 


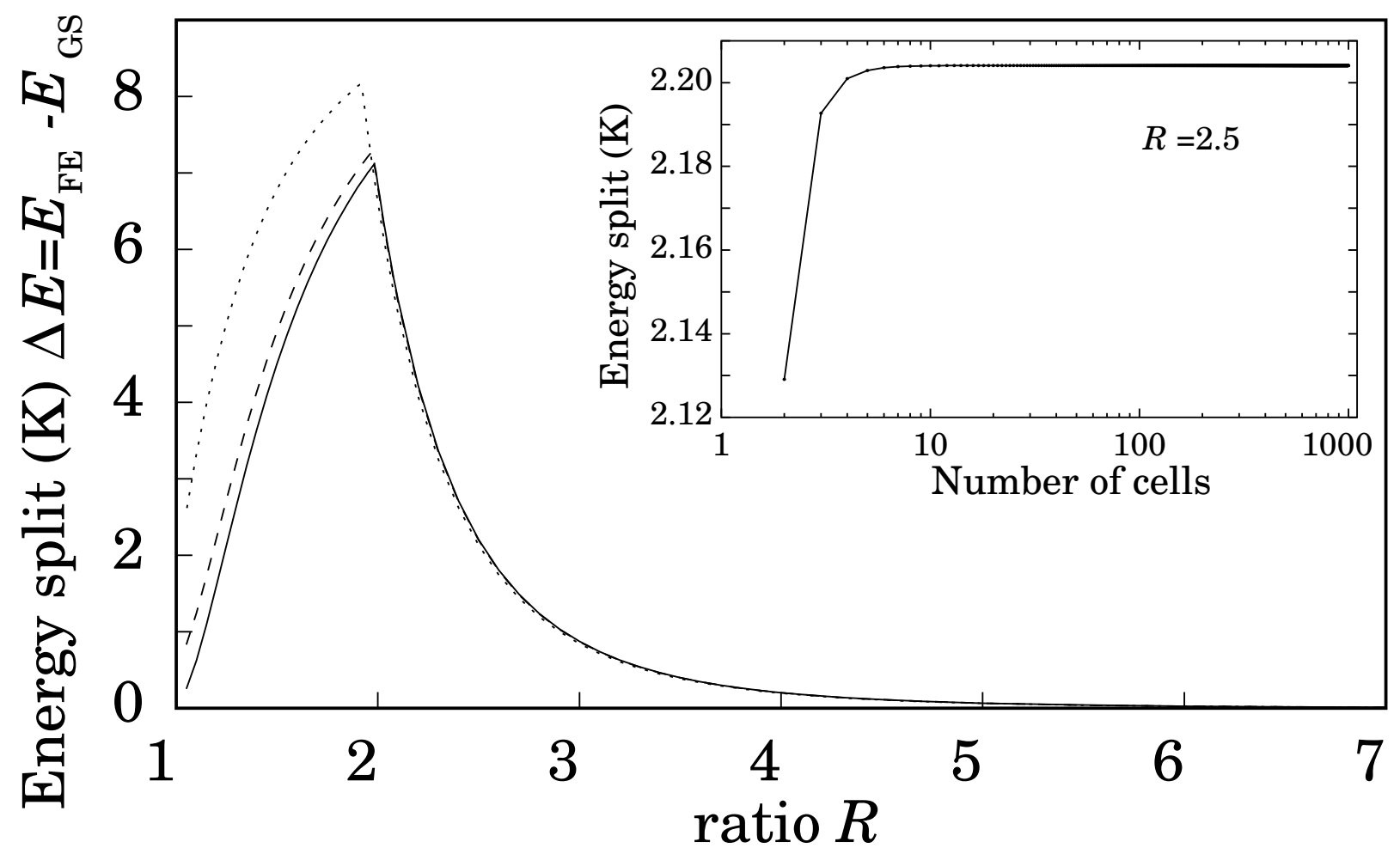

Fig. 3

C. Ungarelli et al. J. Appl. Phys. 


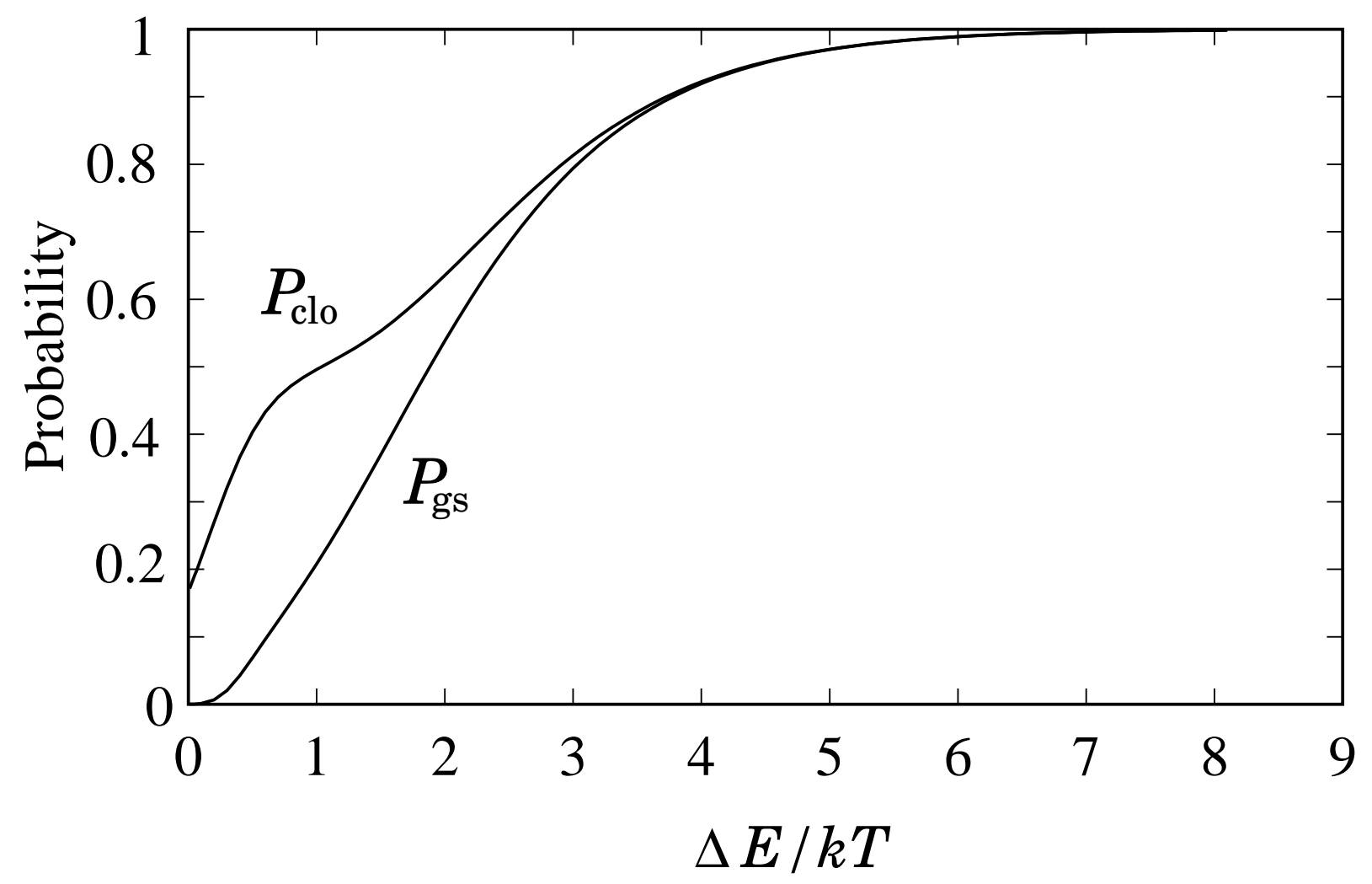

Fig. 4

C. Ungarelli et al.

J. Appl. Phys. 


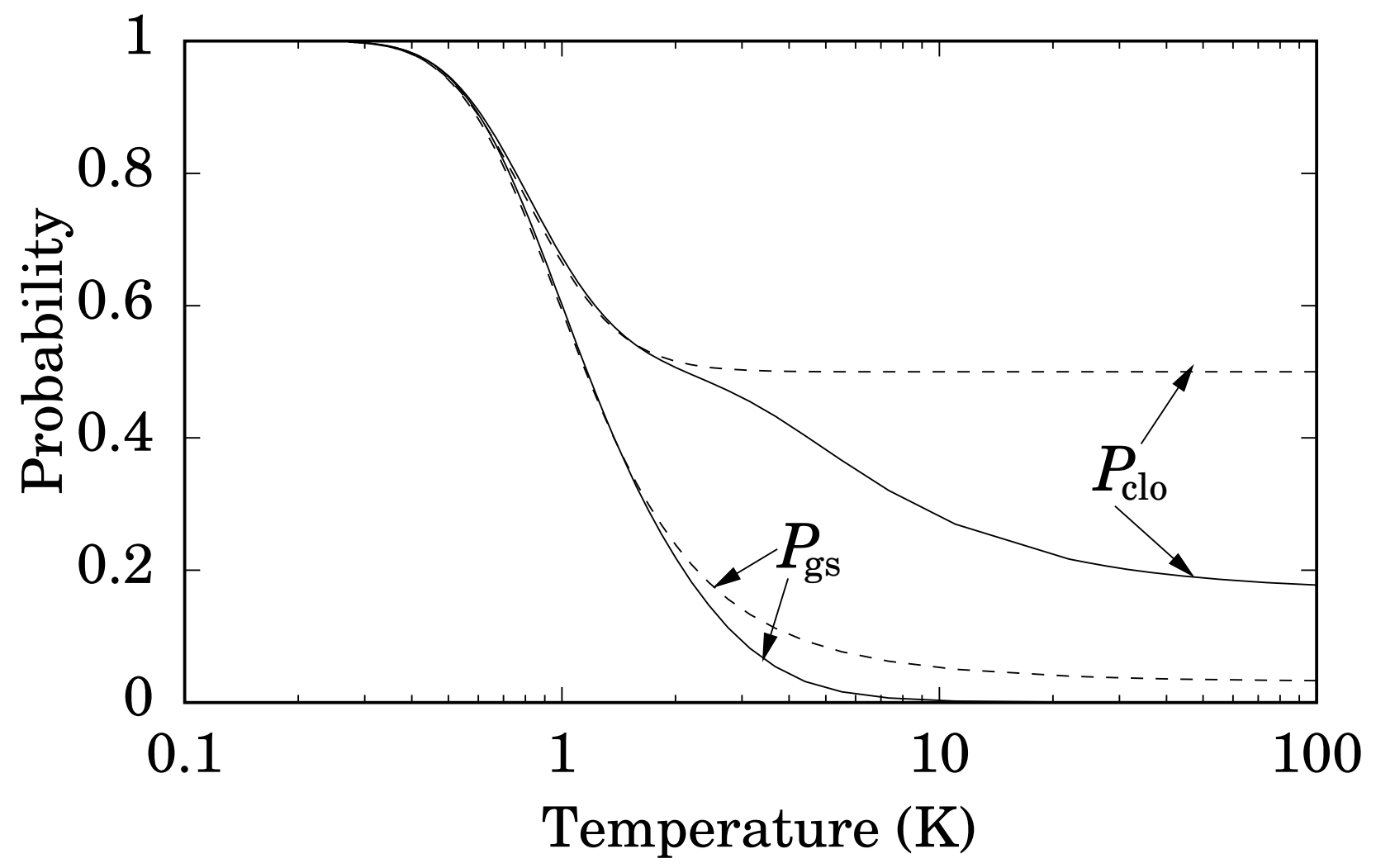

Fig. 5

C. Ungarelli et al.

J. Appl. Phys. 


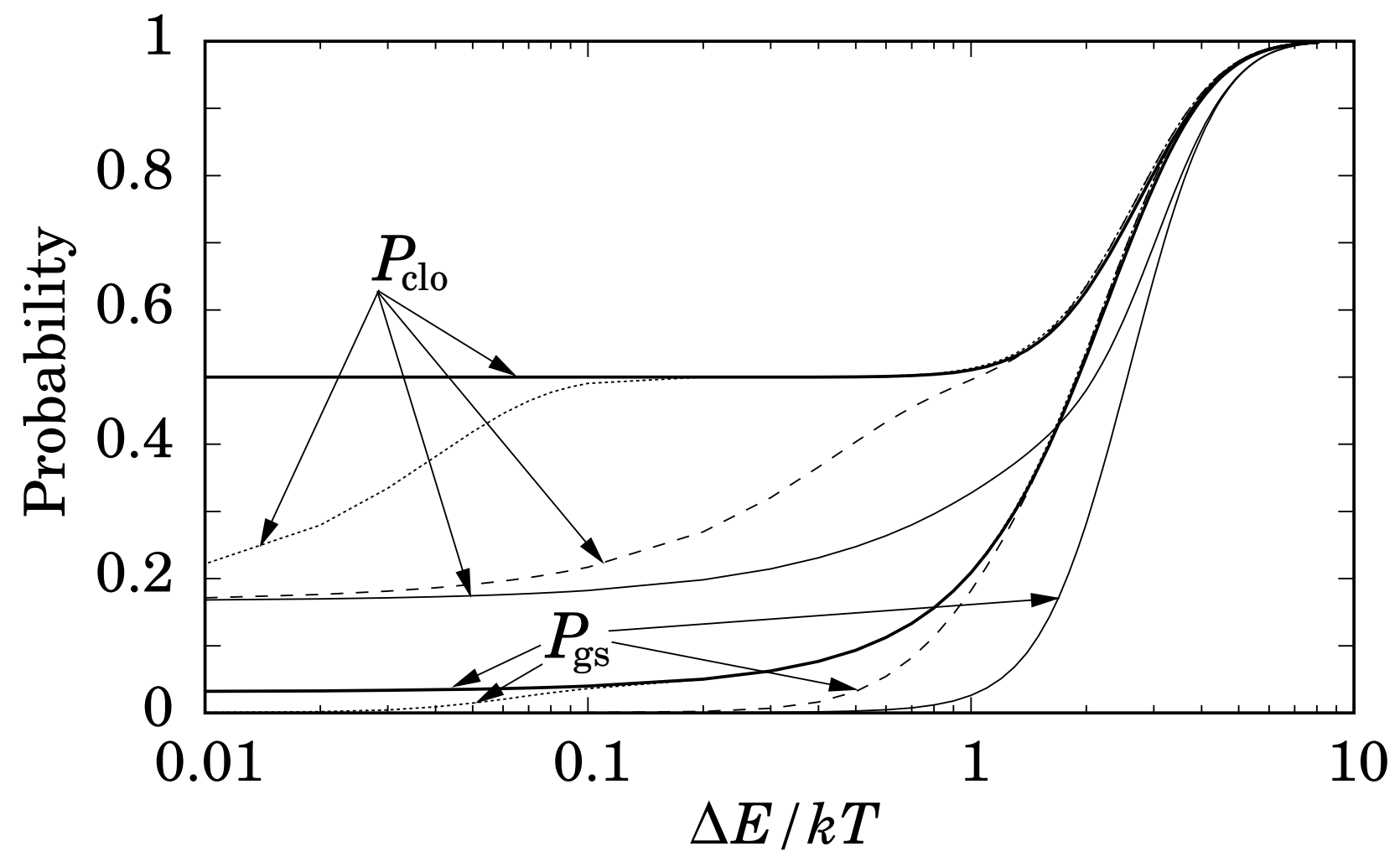

Fig. 6

Ungarelli et al.

J. Appl. Phys. 


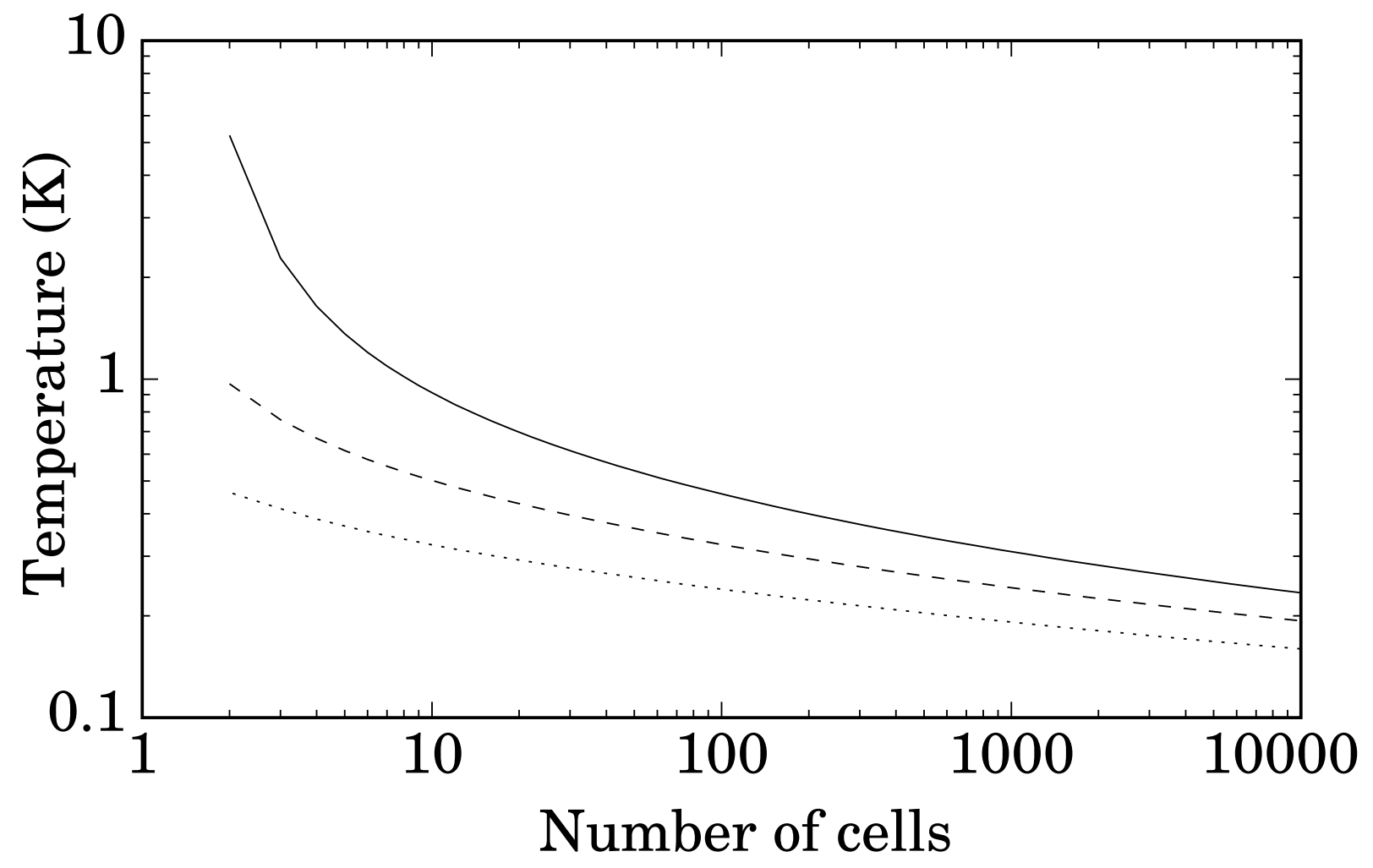

Fig. 7

Ungarelli et al.

J. Appl. Phys. 